\title{
Solar Powered 4-Wheel Drive Autonomous Seed Sowing Robot for Rough Terrain
}

\author{
Fawad NASEER ${ }^{\mathrm{a}, 1}$, Akhtar RASOOL $^{\mathrm{b}}$, M. Zia QAMMAR ${ }^{\mathrm{b}}$ and Rafay SHAHOOD ${ }^{\mathrm{c}}$ \\ ${ }^{a}$ Head of Computer Science Department, Beaconhouse International College, Pakistan \\ ${ }^{\mathrm{b}}$ CS Department Government College University Faisalabad, Pakistan \\ ${ }^{\mathrm{c}}$ CS Department, Beaconhouse International College, Faisalabad, Pakistan
}

\begin{abstract}
In the era of fourth industrial revolution, automation is a growing trend in almost every industry. The innovation in agriculture equipment is one of the major phase for civilized life, and the development of agricultural tools is a fundamental need towards the improvement of agriculture. Farmers use the same traditional methods and equipment for all ages, for example: seeds, spraying, weeding, etc., which have problems such as slow growth rate, irrigation, fertilization, crop monitoring of large areas. An autonomous, low-maintenance and portable robot can serve this purpose more accurately and efficiently with much better performance output. This article introduces the proposed model and design of a solar powered 4wheel drive robot which works to plant seeds while cultivating the soil in a multi terrain surface. The robot avoids human effort ranging from the field path following to uniformly sowing of seeds at equal distance intervals using field area constraints prescribed by the farmer. This article presents the step by steps designing a robot and the parameters to be considered before creating a prototype. This robot increases the efficiency of seeding by exact measurement and distance, flattening of a surface, spraying of initial water, and also reduces random spreading of seeds problems.
\end{abstract}

Keywords. 4-Wheel Robot, Intelligent Farming, Arduino, Seed Sowing, Solarpowered, Agriculture, 4IR.

\section{Introduction}

World population is expected to grow about more than 8 billion by 2035, which will accelerate the global food consumption. To increase yields, the entire planting process needs to be systematically improved to maximize the growing capability to overcome this scenario. Agriculture is adapting automation in agricultural products and field cultivation methods. Subjective processing has become the most practical technology in agricultural services as it allows understanding, studying and responding to different circumstances to improve efficiency. Proximity sensor, humidity sensor and image sensor are different technologies which are mainly used as a judicious combination of information. An example of such high resolution data is soil analysis. Proximity detection requires the sensors to be in contact with the ground or very close. It helps characterize the soil below the surface at a specific location in a field [1]. Generally experienced farmers are familiar with traditional multi-row planting equipment.

1 Fawad Naseer, Head of Computer Science Department, Beaconhouse International College, Recognized Teaching Centre of University of London, 54- West Canal Road, Faisalabad, Pakistan; E-mail: fawad.naseer@bic.edu.pk 
Farmers have following restrictions in normal classical sowing methods [2]:

- Inconsistent distribution of seeds in the corps field.

- Due to Inconsistent distribution of seeds, which will result in gaps between plants.

- Dead seeds due to placement of seeds at incorrect depth of soil.

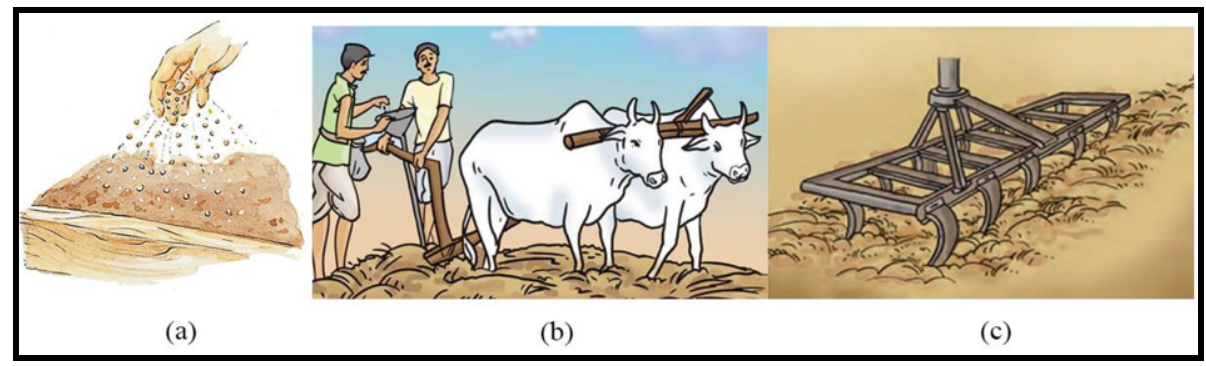

Figure 1. Classical methods of sowing seeds.

Classical methods of sowing and spraying are available in almost all parts of the country, as shown in Fig 1, which results in low yields [3]. Further factors, such as insufficient energy availability and low mechanization rates, also affect crop productivity. Delays in sowing, harvesting, threshing, and improper nursery preparation also reduce crop productivity. Therefore, agricultural modernization cannot be avoided to meet population growth and food needs due to rapid industrialization [3].

Several different agricultural mechanisms have been established to speed up the seed distribution method in the corps field. The manual planter [4] requires farmers to operate and wheel rotation is used to operate the seed release mechanism. In contrast, electric seed growers use diesel engines or electric motors to drive internal mechanisms and are often mounted on tractors. The main drawback of these systems is their low energy efficiency as they require a lot of labor or fossil fuels to operate.

\section{Related Works}

Because there is no effective equipment to support farmers, new technology must be implemented to empower farmers with the capability to ensure and cope up the required demand of global need.

In [5], H. Pota R Eaton, J Katupitiya and S D Pathirana conclude that sowing bulks of seeds is necessary because the number of qualified sowers is greatly reduced. Planting distance and plant population are important factors in maximizing crop yield. By using the microcontroller, 8051 is used for communication between input and output devices. The main disadvantage of this model is that it has only one mechanism.

In [6], authors discussed the mechanisms of automatic tillage, sowing, fertilization and watering. Automatic sowing and fertilization is carried out using solenoids. The soil moisture sensor is used for automatic watering applications using Rasbeery pi and the Internet. The Arduino mega 2560 is used for the robot process and the Rasberry pi is used for communication with the robot. Internet systems are used by farmers to communicate with robots to run processes automatically. It reduces the burden on farmers.

In [7], the development of a GSM-based automatic drip irrigation system was the main focus. The system uses multiple sensors to get the state of the field and the irrigation 
schedule is based on this data. Microcontroller and App / DTMF are used for control and communication respectively. The author has developed a system for planning and scheduling irrigation processes based on real-time information on the spot. Several irrigation issues have been resolved, including the physical labor of farmers to manage irrigation, wasted water and wasted time.

The above research articles have helped us to understand the different aspects induced by research on agricultural robots. The robot designed in the literature search above has many issues with the robot's movement, sowing, power issues and other accuracy concerns. These questions are effectively addressed in this task. This work also sheds light on the future range of robots.

\section{Theory of Seed Sowing}

Sowing seeds is defined as the process of placing seeds under the soil so that it grows to become the plant. For comparison, planting is an acquaintance with the placing the propagules of plants in the soil for growing plants. which can be seedlings, roots, tubers, leaves or cuttings. Seeds may be sown directly or transplanted. The methods of sowing are enlisted and detailed as under:

- Drilling

- Dibbling

- Broadcasting

- $\quad$ Sowing with bamboo plough

As all mentioned above techniques to sow seeds in field, we come to know that conventional sowing has restrictions such as not being able to sow uniformly by hand and insufficient control of sowing depth, and labor demand is high, which results in sowing at uneven depths and have a negative effect on the total grown plants.

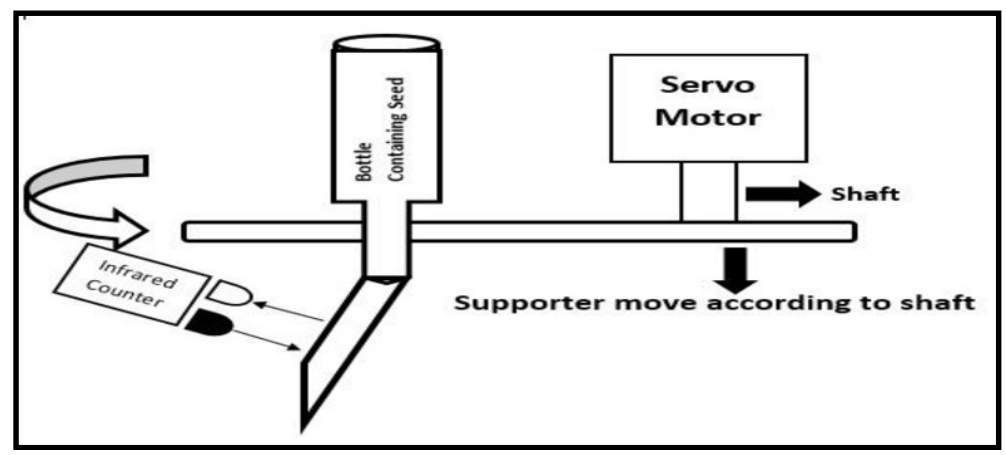

Figure 2. Seed dropping mechanism from seed container along with infrared based seed counter.

The technique which we have develop is that all seeds are collected in a vertical bottle which is mounted on a robot and filled by the farmers as required and dropped using a servo motor to flip the slider and drop seed one by one as shown in Fig. 2. There is an infrared sensor mounted on this mechanism which counts every seed and dropped precisely according to the need of a farmer. Our technique to sow a seed in a soil is the 
drilling mechanism, which is mounted on a robot, it drills the soil and then insert the seed by using the stated mechanism.

\section{Design \& Development of a Proposed Robot}

There are a lot of fields where Artificial Intelligence is impacting human life with better performance results along with less human interaction. The proposed design of our robot is a large scale model to portray working capabilities with better performance including high accuracy of placing a seed under soil and placing seeds with accurate measured distance.

\subsection{Construction}

At initial stage robot mechanical design was developed using computer-aided design (CAD) and later we built it by using steel pipes, which is shown in Figure 3. The cylindrical seed boxes have been chosen to pour the seeds in bulk and for that 1.5-liter PET bottle have been used because they are light, cheap and easy to obtain. The payload consisting of seed boxes and electronic boxes is assigned a maximum weight of $23 \mathrm{~kg}$.

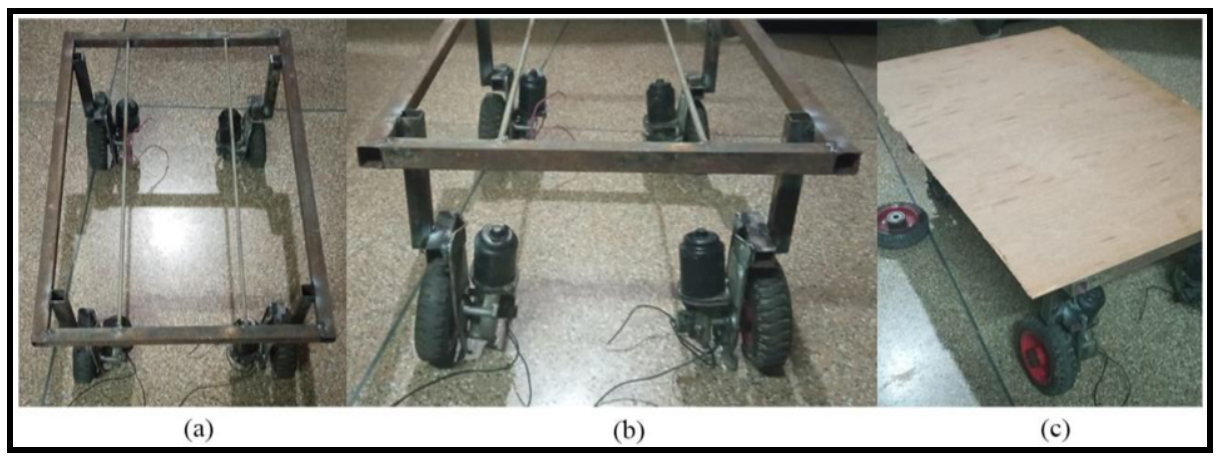

Figure 3. Steel rectangular pipe based robot chassis frame with four dc motors and tires.

We have designed the size of our robot frame as 20-inch $\times 30$-inch $\times 13$-inch to fit the payload frame. The frame is made of steel, which provides strong support for tough terrain, prevents the robot from rolling over, and has the power to effectively control its movements. Each tire is equipped with four DC motors, enabling the robot to move dynamically over rugged terrain and control it in a variety of circumstances at any cost. In the figure 3 shows a drive system consisting of four DC motors connected to all four buses. The diameter of the sprocket connected between the DC motor and bus is determined using a formula:

$$
d=\frac{p}{\sin (180 / z)}
$$

Where $p$ is the pith of the sprocket, $d$ is the diameter of the sprocket and $z$ is the number of teeth on the sprocket. All the variables are decided on the concern of the required speed of the robot to be smooth and stable over different rough terrain. We can extract the dimension of the sprocket to be $93.76 \mathrm{~mm}$ by using the Eqn. (1) by using the number of teeth to be 17 . 
Once we able to get the dimension of the sprocket, from this we can calculate the speed of our robot by using Eqn. (2), where $N$ is the rotation per minute of a sprocket and for this robot we use it to be $200 \mathrm{rpm}$ :

$$
v=\frac{\pi d N}{60}
$$

we can use Eqn. 3 to calculate the required drive power transmitted of all four motors in a robot, each with a stall current of 10A. By using the dimension and velocity which we have calculated and maximum torque to be generalized to be $5 \mathrm{Nm}$ as mentioned on each motor.

$$
P=\frac{2 \pi N T}{60}
$$

The block diagram consists of an Arduino microcontroller, which is the main controller of the entire system along with DC motors, sensors and all helping components as shown in Fig 4. Solar panels connected to the battery for energy storage, as well as a charging circuit that supplies $+5 \mathrm{~V}$ to the Arduino board. Our battery system provides + $12 \mathrm{~V}$ power supply to control the DC motor using the L298 motor driver module.

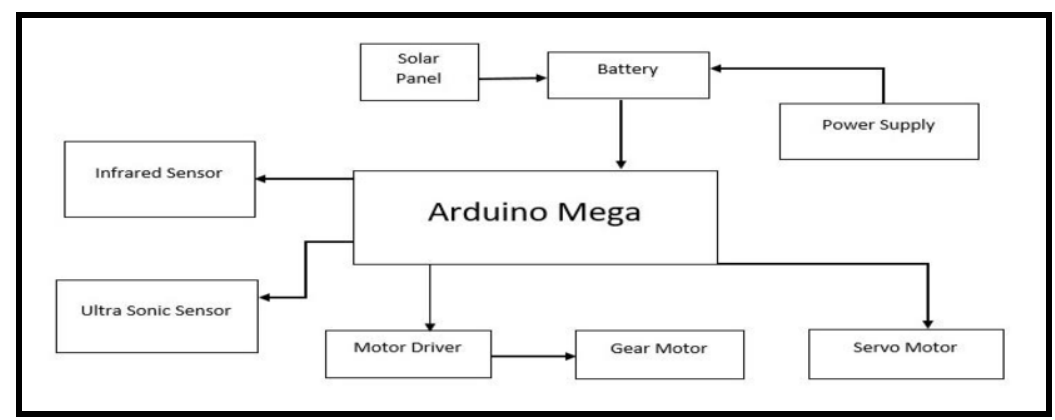

Figure 4. Blok diagram of complete electronic components

\subsection{Electronic Components}

The electronic system of robot consists of a microcontroller and drive circuits required to connect a robot to various mechanical parts as shown in Fig 4. The microcontroller (MCU) is an Arduino Mega 2560, which is the main controller and brain of a complete electronic system. This was chosen because of its high processing power and wide range of peripherals attaching capability, which makes it easy to apply control algorithms. When the robot is placed on the surface of the field, it intelligently orients around the field to cover all areas. The edge of the field was detected using the ultrasonic proximity sensor HC-SR04. This component is used to identify areas that reside on field boundaries [8]. The MCU uses a motor driver connected to a DC motor to control the movement of the robot. Each motor driver consists of a $\mathrm{H}$ bridge power MOSFET connected to the MCU.

\subsubsection{Arduino Mega 2560}

Arduino is an open source physical computing platform based on a simple I / O board and development environment. It is an Atmega 2560 based microcontroller board, which 
is used to interact with all sensors and actuators. It keeps all the components well synchronized for the proper functioning of the robot.

\subsubsection{Motor Driver L298N}

The L298N is a H-bridge driver with high current load. The L298N can supply up to $1 \mathrm{~A}$ at voltages between 4.5 and 36 . The $\mathrm{L} 298 \mathrm{~N}$ is designed to drive inductive loads such as solenoids, relays, DC motors, bipolar stepper motors, and other powerful motors. application of current or high voltage.

\subsubsection{Ultrasonic Sensor HC-SR04}

HC-SR04 ultrasonic sensor is used to calculate the distance to an object using sonar signal. It uses a non-contact ultrasonic sounder to measure the distance to an object and consists of two ultrasonic transmitters (mainly loudspeakers), a receiver and a control circuit [9]. The transmitter emits high-frequency ultrasonic waves that are reflected off nearby solids, and the receiver tracks the reflected echo. This echo is then processed by the control circuit to calculate the time difference between the transmit and receive signals [10].

\subsubsection{Servo Motor}

Servo motors are half-turn actuators used in applications that require a high degree of swing angle accuracy. This is because the servo motor has a feedback sensor. The feedback sensor measures the difference between the set and the required angle to ensure that you reach the end position more accurately.

\subsubsection{Power Source}

A battery is a source of energy that provides a surge (voltage) of energy to circulate current in a circuit. It powers the entire circuitry including all the components attached to the system. Our entire system is powered by a pair of $18650 \mathrm{Li}$-ion batteries through the battery management system(BMS) [11]. Sonar sensors have a built-in voltage regulator; hence it is powered from any DC voltage from 5 volts. Modules are a group of cells that are electrically connected and packaged in a frame (most commonly known as a solar panel).

\section{Functioning of The Proposed Robot}

The working principle of the developed system is described as a flowchart in Fig. 5. The code is developed to enable the robot to move in all directions which is being controlled intelligent by the microcontroller Arduino Mega 2560 to automate the whole process of seeding. 


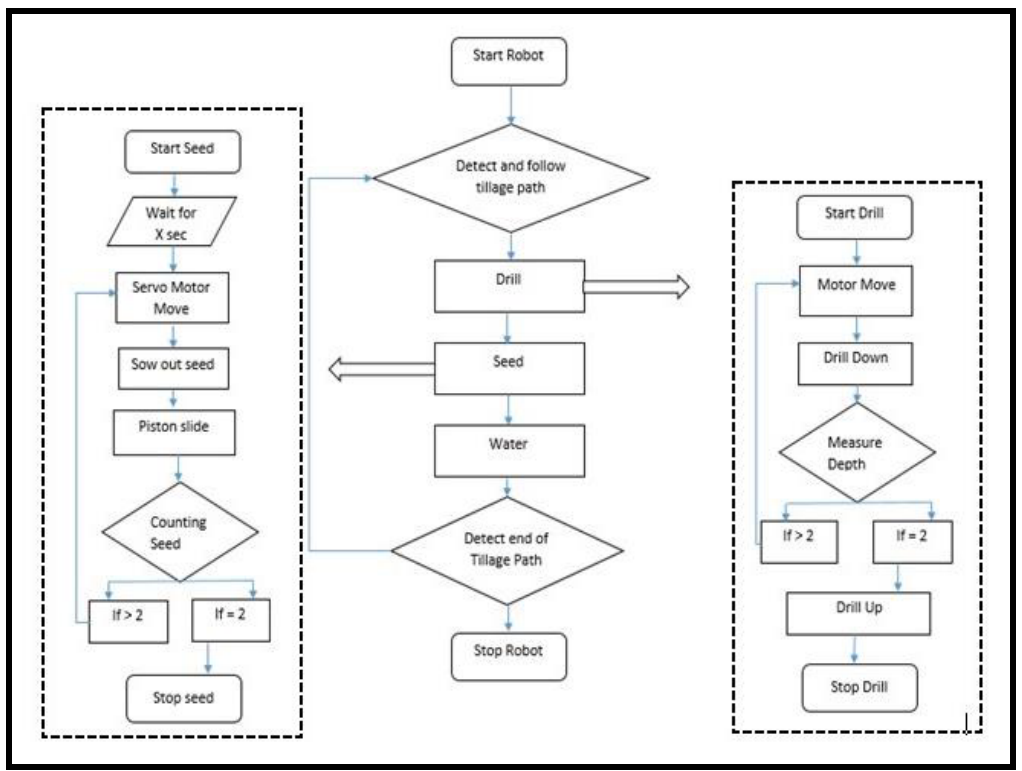

Figure 5. Flowchart of functioning of proposed robot

The robot starts to move forward and the seeds are planted evenly at an equal distance. The seeds are stored in a tapered seed bottle, so the seeds can easily enter the funnel through the attached hose. The seed placement mechanism runs smoothly and uses servo motors to complete the seeding tasks. It consists of two-stroke sliders used to control the opening of the hole from which the seeds are poured. After each drop of a seed, there is an infrared sensor which is used to count the number of seeds passed through the funnel to the hole. After pouring of pair of seeds, water sprinkle is placed, its duty is to sprinkle water on that sowed seed. After watering task is to flat the surface and for that a flap which is attached behind the robot to cover the soil as shown in Fig 6.

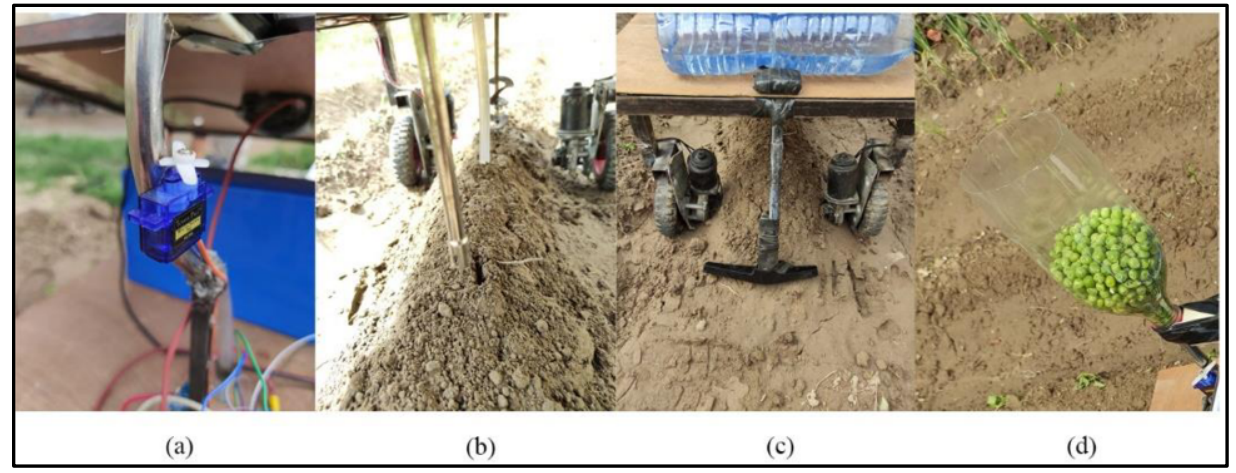

Figure 6. (a) Seeding drop mechanism, (b) Water sprinkler, (c) Flapper to flap surface \& (d) Seed container.

When the robot deviates from the intended direction of motion due to undulations in the tillage path, the input provided by the ultrasonic sensor varies. This aberration is reduced by using the microcontroller which alters the power of the individual motors as described in Table 1. Thus the controller allows the device to traverse in the correct direction. 
Table 1. Motor direction controlling algorithm

\begin{tabular}{ccccc}
\hline Movement Type & Motor A(Left Front) & Motor B(Left Rear) & Motor C(Right Front) & Motor D(Right Rear) \\
\hline Straight & ON & ON & ON & ON \\
Left & OFF & OFF & ON & ON \\
Right & ON & ON & OFF & OFF \\
\hline
\end{tabular}

Motor A and B are on left side of a robot and Motor C and D are on right hand side of a robot. Ultrasonic sensors are used to determine the end of the field and, when detected, rotate the robot in different directions of travel. This allows the robot to plant seeds in subsequent rows that are zigzag known as tillage rows as shown in Fig 7.

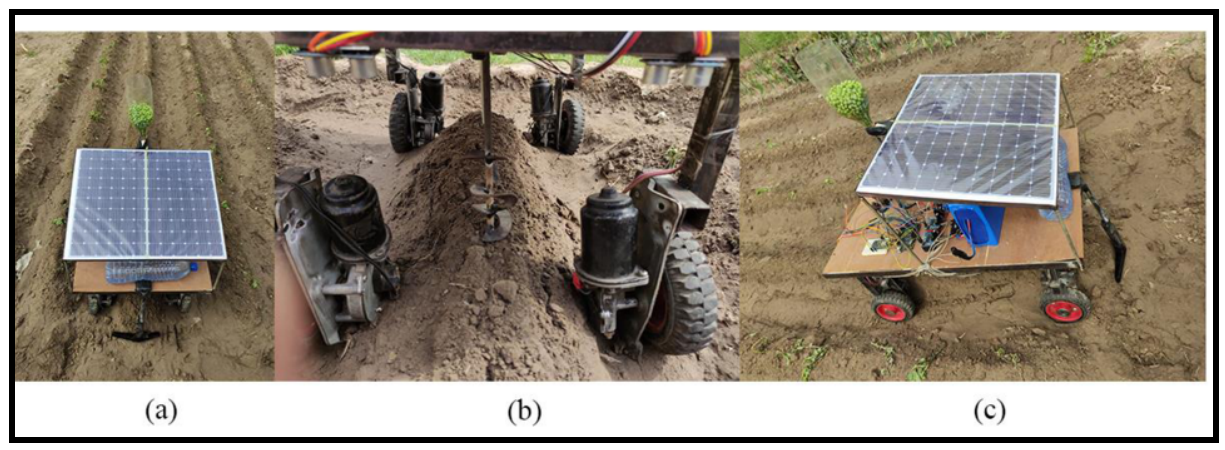

Figure 7. (a) Top view of robot, (b) Front bottom view of robot \& (c) Side view of robot.

\section{Results and Discussion}

In the first series of experiments which was performed to validate the possibility to drill the accurate required depth to pour a pair of pea seeds to germinate. In this experiment, the planting testbed replicated the field environment which comprises of soil. The drill was prepared so that the precisely hole can be achieved at precise depth and pouring of 2 seeds as a pair in that hole. The prepared drill was prepared and dig into soil and waited for 30 days after successfully sowing of seeds. The moisture in the soil would dissolve the seeds entirely. All seeds inside a hole successfully germinated into pea cotyledon which is shown in Fig. 8.

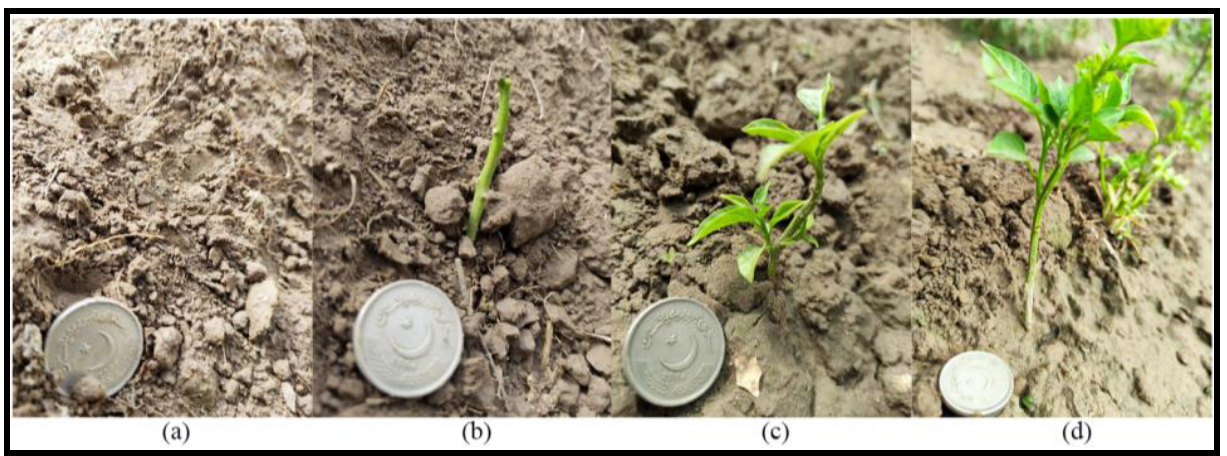

Figure 8. Seed germination after drilling hole from a robot. 
In the second series of experiments, we have tried to sow different types of seeds and analyze required depth for each seed and the required spacing between sowing of seeds in field, we also analyze the size of different seeds and come to know that each seeds with different size required different size of digging hole for sowing, but our robot at current state is not able to dig a customized hole according to the seed, it ca be a future work to implement. Table 2 shows the comparison of the types of seeds, their dimension, required spacing of sowing and the required depth.

Table 2. Seed Analysis and Comparison

\begin{tabular}{cccccc}
\hline Type of seed & Length(mm) & Width $(\mathbf{m m})$ & Diameter(mm) & Spacing(inch) & Depth(inch) \\
\hline Pea & 5.91 & 5.01 & 5.18 & $5-7.5$ & 5 \\
Tomato & 6.3 & 6.9 & 4.23 & $6.5-8$ & 5 \\
Sun Flower & 9.52 & 5.12 & 3.27 & $6-7$ & 3 \\
Chia & 2.11 & 1.3 & 0.8 & $3.5-5$ & 4 \\
Flax & 4.64 & 2.37 & 1.00 & $4.5-6$ & 5
\end{tabular}

Figure 9 shows the graph of approximation of dropping seeds inside the hole dig by the drill in the different experiments we did before installing that seeding part on the robot. The graph explains that number of times the dropping of seeds and its accuracy to drop exactly inside the hole or scattering around the hole.

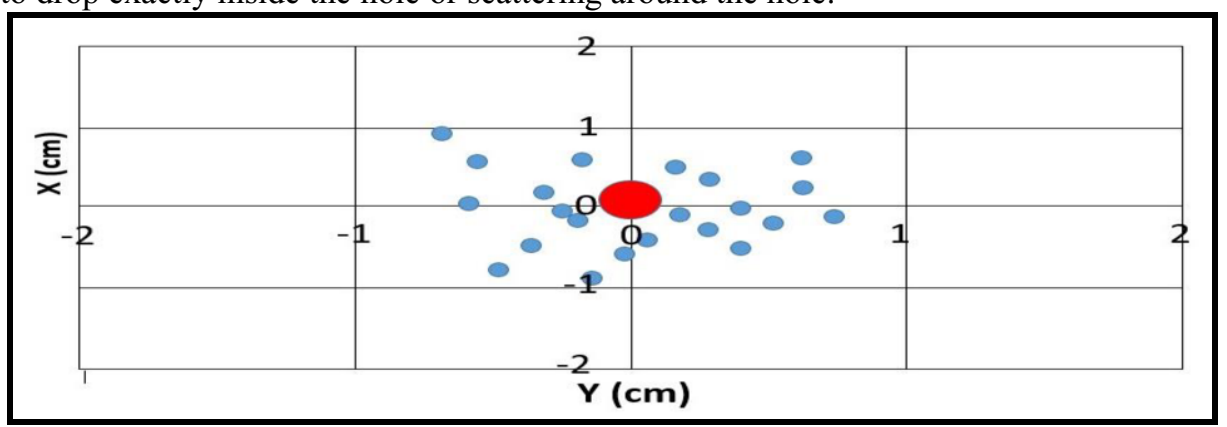

Figure 9. Experiment result of conducting different times seed dropping in hole.

\section{Conclusion and Future Work}

The design and development of a robot along with the Working procedure of the autonomous seed sowing mechanism have been proposed for the sole purpose of which is to automate the seed sowing process with flattening of the surface and watering over newly sowed seeds is discussed in this paper. All components except the sensors are individually designed, and the entire seed mechanism workflow and sequence of executions are illustrated in the block diagram above. Different models of seed collectors have also been developed, depending on the type of seed.

For the future purpose, the robot can be equipped with Global Positioning System (GPS) for more accuracy of the movement of robot and also for future perspective, robot can be equipped with more powerful processing board which can be used to calculate intelligent algorithm including machine learning algorithm for better performance of robot in context of accuracy in navigation around the field. 


\section{Acknowledgment}

We would like to thank our respected Country Head Ms Raana Sarmad, for the inspiration and motivation towards creating a research culture in undergraduate students.

\section{References}

[1] PJ, S. K., "A brief survey of classification techniques applied Sheela to soil fertility prediction", International Conference on Emerging Trends, 2015.

[2] Pratik Hore. a, Goluprasad Gupta. a, Shrikrishn Deshmukh, "Modern seed sowing techniques and developing technologies," International Journal of Innovative and Emerging Research in Engineering, Volume 4, Issue 3, 2017.

[3] D. Ramesh, H.P. Girishkumar, Agricultural Seed Sowing Equipments: A Review International Journal of Science, Engineering and Technology Research (IJSETR), Volume 3, Issue 7, July 2014

[4] D. Ramesh and H.P. Girishkumar, "Agriculture Seed Sowing Equipments : A Review", International Journal of Science, Engineering and Technology Research (IJSETR), vol. 3, Issue 7, July 2014, pp. 1987 - 1992.

[5] H. Pota, R. Eaton, J. Katapriya and S. D. Pathirana, "Agricultural robotics: A streamlined approach to realization autonomous farming," in IEEE conference on industrial and information systems, 2007, pp. 85-90.

[6] Gollakota, A. and Srinivas, M.B., (2011), December. "Agribot - a multipurpose agricultural robot", In India Conference (INDICON), 2011 Annual IEEE (pp. 1-4). IEEE

[7] Lalwani, A., et al., 2015, December, “A Review: Autonomous Agribot For Smart Farming”, Proceedings of 46th IRF International Conference, 27th December 2015, Pune, India

[8] J. Borenstein and Y. Koram, "Obstacle Avoidance with Ultrasonic Sensors", IEEE Journal of Robotics and Automation, vol. 4, no. 2, April 1988, pp. 213-218.

[9] S. Barai, D. Biswas and B. Sau, "Estimate distance measurement using NodeMCU ESP8266 based on RSSI technique," 2017 IEEE Conference on Antenna Measurements \& Applications (CAMA), Tsukuba, 2017, pp. 170-1

[10] F. Noor, M. Swaied, M. AlMesned and N. AlMuzini, "A Method to Detect Object's Width with Ultrasonic Sensor," 2018 International Conference on Computing, Electronics \& Communications Engineering (iCCECE), Southend, United Kingdom, 2018, pp. 266-271

[11] Khanna,A;Ranjan, "Solar-powred Android based Speed Control of DC motors through Secure Bluetooth," Communication systems and network technologies CSNT 2015 international conference (IEEE Publication), pp 1244-1249.

[12] P. Amiribavandpour, A. Kapoor, W. Shen and J. Shearer, "Thevmathematical model of 18650 lithiumion battery in electric vehicles," 2013 IEEE 8th Conference on Industrial Electronics and Applications (ICIEA), Melbourne, VIC, Australia, 2013, pp. 1264-1269 